\title{
Water Networks Contribute to Enthalpy/Entropy Compensation in Protein-Ligand Binding
}

\section{Citation}

Breiten, Benjamin, Matthew R. Lockett, Woody Sherman, Shuji Fujita, Mohammad Al-Sayah, Heiko Lange, Carleen M. Bowers, Annie Heroux, Goran Krilov, and George M. Whitesides. 2013. "Water Networks Contribute to Enthalpy/Entropy Compensation in Protein-Ligand Binding." Journal of the American Chemical Society 135(41): 15579-15584.

\section{Published Version}

doi:10.1021/ja4075776

\section{Permanent link}

http://nrs.harvard.edu/urn-3:HUL.InstRepos:12361263

\section{Terms of Use}

This article was downloaded from Harvard University's DASH repository, and is made available under the terms and conditions applicable to Open Access Policy Articles, as set forth at http:// nrs.harvard.edu/urn-3:HUL.InstRepos:dash.current.terms-of-use\#OAP

\section{Share Your Story}

The Harvard community has made this article openly available.

Please share how this access benefits you. Submit a story.

\section{Accessibility}




\title{
Water Networks Contribute to Enthalpy/Entropy Compensation in Protein-Ligand Binding
}

\author{
Benjamin Breiten,,${ }^{a, \hbar}$ Matthew R. Lockett, ${ }^{a, t}$ Woody Sherman, ${ }^{b}$ Shuji Fujita, ${ }^{a}$ Mohammad Al- \\ Sayah, ${ }^{a}$ Heiko Lange, ${ }^{a}$ Carleen M. Bowers, ${ }^{a}$ Annie Heroux, ${ }^{c}$ Goran Krilov, ${ }^{b}$ and George M. \\ Whitesides ${ }^{\mathrm{a}, \mathrm{d}, *}$ \\ ${ }^{a}$ Department of Chemistry and Chemical Biology, Harvard University \\ 12 Oxford Street, Cambridge, MA 02138 \\ ${ }^{b}$ Schrödinger, Inc., \\ 120 West $45^{\text {th }}$ Street, New York, NY 10036-4041 \\ ${ }^{c}$ National Synchrotron Light Source, Photon Sciences Directorate \\ Building 745, Brookhaven National Laboratory, Upton, NY 11973-5000 \\ ${ }^{d}$ Wyss Institute for Biologically Inspired Engineering, Harvard University \\ 60 Oxford Street, Cambridge, MA 02138
}

\$ These authors contributed equally to this work

* Author to whom correspondence should be addressed. 


\begin{abstract}
The mechanism (or mechanisms) of enthalpy-entropy $(H / S)$ compensation in protein-ligand binding remains controversial, and there are still no predictive models (theoretical or experimental) in which hypotheses of ligand binding can be readily tested. Here we describe a particularly well-defined system of protein and ligands - human carbonic anhydrase (HCA) and a series of benzothiazole sulfonamide ligands with different patterns of fluorination - that we use to define enthalpy/entropy $(H / S)$ compensation in this system thermodynamically and structurally. The binding affinities of these ligands (with the exception of one ligand, in which the deviation is understood) to HCA are, despite differences in fluorination pattern, indistinguishable; they nonetheless reflect significant and compensating changes in enthalpy and entropy of binding. Analysis reveals that differences in the structure and thermodynamic properties of water surrounding the bound ligands are an important contributor to the observed $H / S$ compensation. These results support the hypothesis that the molecules of water filling the active site of a protein, and surrounding the ligand, are as important as the contact interactions between the protein and the ligand for biomolecular recognition, and determining the thermodynamics of binding.
\end{abstract}




\section{Introduction}

The hydrophobic effect- the tendency of nonpolar molecules or parts of molecules to aggregate in aqueous media - is central to biomolecular recognition. It now seems that there is no single "hydrophobic effect"1-4 that adequately describes the partitioning of a small apolar ligand between both i) an aqueous phase and a non-polar organic phase (e.g., buffer and octanol), and ii) bulk aqueous buffer and the active site of a protein (i.e., biomolecular recognition). While the molecular-level mechanisms of hydrophobic effects in biomolecular recognition remain a subject of substantial controversy, it is clear that the water molecules surrounding the apolar ligand and filling the active site of the protein are an important part of these mechanisms. ${ }^{1-10}$ Clarifying the role of water in the hydrophobic effect in protein-ligand binding would be an important contribution to understanding the fundamental, mechanistic basis of molecular recognition. Resolving this mechanism would, however, still leave a (presumably) related phenomena unresolved: so-called, enthalpy-entropy compensation ( $H / S$ compensation). $H / S$ compensation is often encountered in the putative design of tight-binding, lowmolecular-weight ligands for a protein. ${ }^{11,12}$ Changes in the structure of the ligand often lead to opposite and compensating changes in the enthalpy and entropy of binding, but result in surprisingly small changes in the free energy of binding. The molecular-level mechanism of $H / S$ compensation in protein-ligand binding - and even its existence as a phenomenon ${ }^{12}$ - remains a subject of substantial controversy — even at a conceptual level—despite qualitative rationalizations based upon: i) an unfavorable entropy of binding caused by conformational restrictions of the ligand upon binding; ${ }^{13-15}$ ii) small conformational changes throughout the protein upon ligand binding; ${ }^{16,17}$ iii) reorganization of solvent molecules within the active site of a protein after ligand binding. ${ }^{18-20}$ 
Human carbonic anhydrase II (HCA, EC 4.2.1.1) is an excellent model system with which to study $H / S$ compensation because it allows us to answer the question: "Do changes in the structure of the networks of hydrogen bonded waters - that result upon ligand binding determine $H / S$ compensation?". Thiazole-based sulfonamide ligands bind to HCA with an enthalpy-dominated hydrophobic effect, ${ }^{2,4}$ and are not compatible with an entropy-dominated hydrophobic effect proposed by Kauzmann and Tanford. ${ }^{21,22}$ or the $H / S$ compensation proposed by Dunitz, Williams, and others. ${ }^{13-15} \mathrm{HCA}$ is conformationally rigid and undergoes minimal $(<1$ A) conformational changes upon binding of most arylsulfonamide ligands ${ }^{23}$-and, more importantly for this study thiazole-based sulfonamide ligands ${ }^{2,4}$-and allows us to focus solely on rearrangements of solvent within the active site of the protein, and not on contributions caused by conformational changes in the protein.

We have measured changes in the thermodynamics of binding of a series of heteroarylsulfonamide ligands to HCA with isothermal titration calorimetry (ITC) in which a benzo-, fluorobenzo-, or tetrahydrobenzo-group was added to one edge of the ligand (Figure 1A). ${ }^{2,4}$ Remarkably, the free energies of binding ( $\Delta G^{\mathrm{o}}$ bind $)$ of benzothiazole sulfonamide $\left(\mathbf{H}_{4}\right.$ BTA $)$ and perfluorobenzothiazole sulfonamide $\left(\mathbf{F}_{4} \mathbf{B T A}\right)$ to HCA are indistinguishable, ${ }^{2}$ and suggests that the hydrophobic effects in this protein-ligand system are insensitive to this substantial change in the chemical composition and electronic structure of the ligand. While the values of $\Delta G^{\mathrm{o}}$ bind of $\mathbf{H}_{4}$ BTA are unchanged upon fluorination, we do observe significant and compensating changes in the enthalpy ( $\Delta H^{\mathrm{o}}$ bind $)$ and entropy ( $-\mathrm{T} \Delta S^{\mathrm{o}}$ bind $)$ of binding, ${ }^{2}$ indicating that the underlying mechanism of molecular recognition may be different even though the total free energy of binding is the same. 
ITC thermograms of protein-ligand binding contain three values - the binding affinity of the ligand, the $\Delta H^{\circ}$ bind, and the stoichiometry of binding — that are obtained from a non-linear fit of the data. Uncertainty in the concentration of the ligand is the main source of measurement error in ITC, and leads to errors in $K_{\mathrm{a}}$ and $\Delta H^{\mathrm{o}}$ bind - as well as $\Delta G^{\mathrm{o}}$ bind and $-\mathrm{T} \Delta S^{\mathrm{o}}$ bind, which are calculated from these values. ${ }^{12,24}$ In order to reduce artifacts in our ITC measurements that could lead to perceived $H / S$ compensation we: i) measured the binding of a standard sulfonamide (methazolamide), whose concentration was determined accurately with NMR standards, to HCA and obtained the concentration of active protein; ii) prepared stock solutions of each ligand, and used these stocks solutions for each experiment to eliminate changes in the concentration of the ligand between experiments; iii) compared the binding stoichiometry of each ligand with the methazolamide standard to obtain an accurate concentration of each ligand; iv) accounted for the uncertainties associated with the nonlinear fits used to analyze the thermograms ( $\Delta H^{0}$ bind less than $\sim 10 \%$ ), and compared the average value of ( $\mathrm{n}=7$ runs) each ligand with a Student's t-test with a $95 \%$ confidence interval.

In this paper we wished to determine if selectively replacing the hydrogen atoms of the benzothiazole moiety with fluorine atoms would change the network of waters in the active site of $\mathrm{HCA}$, and result in an $H / S$ compensation similar to that observed between $\mathbf{H}_{\mathbf{4}} \mathbf{B T A}$ and F $_{4}$ BTA. Selective replacement of the C-H bonds of the benzo-group with C-F bonds allow us to study the binding of a set of ligands that are similar in size, but have entirely different group dipole moments and therefore different interactions with the networks of water in the active site. 


\section{Results and Discussion}

Fluorination of the benzothiazole ligand changes its electronic structure, but leaves the size of the ligand and its binding geometry relatively unchanged. Replacing all of the $\mathrm{C}-\mathrm{H}$ bonds of the benzo-extension of $\mathbf{H}_{4}$ BTA with $\mathrm{C}-\mathrm{F}$ bonds results in a decrease of the average dipole moment, but does not result in large changes in: i) the $\mathrm{pKa}$ of the ligand (a decrease of 0.3 units); ii) the solvent accessible surface area of the ligand in crystal structures of the HCA: ligand complex (an increase of $34 \AA^{2}$ ); iii) the binding geometry of the ligands (a shift of $0.7 \AA$ in the binding pocket toward the hydrophobic wall). ${ }^{2}$

We compared the binding geometry of six of the partially fluorinated ligands (4-F $\mathbf{B}$ BTA, 7-

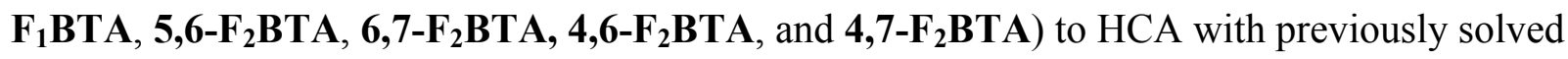
structures of HCA complexed with $\mathbf{H}_{4} \mathbf{B T} \mathbf{A}^{4}$ and $\mathbf{F}_{4} \mathbf{B T} \mathbf{A}^{2}$. Each of the high-resolution crystal structures (Figure 2, resolutions ranging from 1.25-1.50 $\AA$ ) show that a fluorine atom in the 4position causes the ligands to: i) bind to HCA with the same geometry as $\mathbf{F}_{4} \mathbf{B T A}$; ii) rotate $180^{\circ}$ around the molecular axis of the sulfonamide-benzothiazole bond when there is not a fluorine in the 7-position. We attribute these changes in the position of the partially fluorinated ligands to a repulsive interaction between the fluorine atom of the ligand and Thr 200 (Figure 1C).

Surprisingly, the position of the amino acids lining the active site of HCA are not affected by the position or orientation of the ligand: the average root-mean square deviation (RMSD) of the heavy atoms of the amino acids lining the active site of all of the partially-fluorinated ligand with $\mathrm{H}_{4}$ BTA and F $_{4}$ BTA is $0.132 \AA$ and $0.112 \AA$, respectively.

These crystal structures suggest that the interactions between the benzothiazole ligands and the binding pocket of HCA are mediated by the molecules of water in the active site, and not through the traditional lock-and-key model of direct interactions between protein and ligand. 
The binding affinity of benzothiazole is relatively unaffected by fluorination, and in certain cases is the result of compensating values of enthalpy and entropy of binding. We measured the enthalpies of binding ( $\Delta H^{\mathrm{o}}$ bind $)$ and the association constants $\left(K_{\mathrm{a}}\right)$ for the series of the partially fluorinated ligands in Figure 1A with isothermal titration calorimetry (ITC), and estimated the free energies ( $\Delta G^{\mathrm{o}}$ bind) and entropies ( $-\mathrm{T} \Delta S^{\mathrm{o}}$ bind) of binding. Figure 3 plots the $\mathrm{p} K_{\mathrm{a}}$-corrected values of $\Delta J^{0}$ bind for each ligand (where $\Delta J=\Delta G, \Delta H$, or $-\mathrm{T} \Delta S$ ); these values represent the binding of the sulfonamide anion to HCA (details in the SI). ${ }^{4,25}$ We classified the ligands into three categories: i) ligands in which $\Delta H^{\mathrm{o}}$ bind and $-\mathrm{T} \Delta S^{\mathrm{o}}{ }_{\text {bind }}$ are unchanged, and result in an

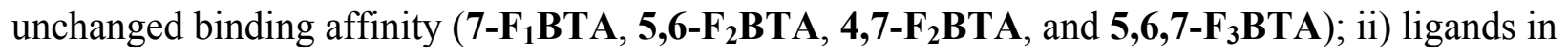
which $-\mathrm{T} \Delta S^{\mathrm{o}}$ bind is significantly different, and results in an increase in binding affinity $(\mathbf{6 , 7 -}$ F $_{2}$ BTA); iii) ligands in which $\Delta H^{0}$ bind and $-\mathrm{T} \Delta S^{\mathrm{o}}$ bind are significantly different, but compensate and result in an unchanged binding affinity $\left(4-F_{1}\right.$ BTA and $4,6-F_{2}$ BTA $)$. A change is considered significant if the values of $\Delta J^{0}$ bind are statistically distinguishable from $\mathbf{H}_{4}$ BTA at $p<0.05$ (by Student's t-test).

\section{Molecular dynamics simulations support the role of water in H/S compensation. Our} previous studies of HCA-arylsulfonamide ligand complexes support the hypothesis that the network of waters within the active site of HCA is an integral component in enthalpically driven hydrophobic effects. Structural and thermodynamic data of the partially fluorinated ligands binding to HCA suggest that the network of hydrogen-bonded water molecules in the active site of a protein-ligand complex could be responsible for changes in the $\Delta H^{\mathrm{o}}$ bind and $-\mathrm{T} \Delta S^{\mathrm{o}}$ bind, but there are currently no experiments to probe the thermodynamic characteristics of individual water molecules directly in the active site of a protein. X-ray and neutron-diffraction data provide information about some of the so-called "fixed" water molecules, but these techniques 
cannot capture the location of the majority of the waters in the active site (before or after the ligand binds). ${ }^{3,26,27}$ In addition, a water molecule that diffracts simply implies that it is spatially immobilized but does not provide information about the energetics of the water.

To understand the thermodynamics of the network of waters in the active site of each HCAligand complex better we performed WaterMap calculations (see Figure 4), ${ }^{9,28,29}$ which are explicit solvent calculations that determine a free energy, enthalpy, and entropy value for each molecule of water in the active site of the protein-ligand complex ${ }^{2,4}$ using an approach called inhomogeneous solvation theory. ${ }^{30,31}$ These calculations support the $H / S$ compensation we observe in the ITC measurements of HCA-ligand binding, and show: i) 4-F $\mathbf{1}$ BTA and 4,6F $_{2}$ BTA have the most favorable entropy of binding, and the least favorable enthalpy of binding. These effects result from subtle disruptions in the network of waters in the solvent-exposed region of the binding site, as seen in Figure 5 (the 4-F $\mathbf{B}$ BA results look highly similar to 4,6$F_{2}$ BTA and are therefore omitted for clarity). The fluorination pattern of these ligands apparently disrupts the water network in a way that results in less restricted water motion and therefore better entropy. ii) WaterMap calculates $5,6-\mathbf{F}_{2}$ BTA to have the least favorable entropy of binding, a result that is also consistent with the experiment. The 5- and 6-positions of the benzoextension are directed toward bulk solvent, and the difluoro substitution is predicted to restrict the mobility of a local region of waters around the ligand. Figure 5 shows an additional hydration site localized above the 5,6-F $\mathbf{2}$ BTA ligand (denoted by a black arrow). WaterMap predicts an

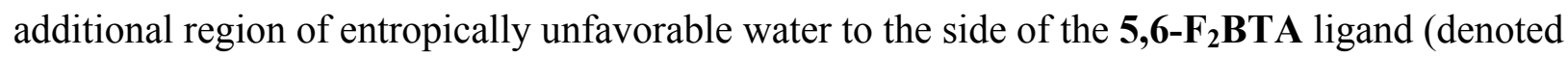
by the dashed oval), further contributing to the unfavorable entropy of binding. iii) $6,7-F_{2} \mathbf{B T A}$

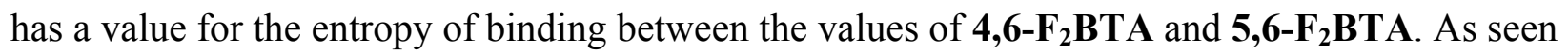
in Figure 5, the region to the right of the ligand (see dashed oval) is similarly unfavorable to 5,6- 
F $_{2}$ BTA, but the region extending toward bulk solvent does not have the additional localized hydration site observed in $\mathbf{5 , 6 - F _ { 2 }}$ BTA (see hydration site indicated by the black arrow in 5,6F $_{2}$ BTA that is missing from $6,7-F_{2}$ BTA)

The improved free energy of binding of $6,7-\boldsymbol{F}_{2} \boldsymbol{B T A}$ (compared to $\boldsymbol{H}_{4} \boldsymbol{B} \boldsymbol{B} \boldsymbol{A}$ ) arises from desolvation of the ligand upon binding, and most likely is not from a rearrangement of the solvent in the active site of $H C A, \mathbf{6 , 7}-\mathbf{F}_{2} \mathbf{B T A}$ is the only ligand that binds to HCA with a higher binding affinity than $\mathbf{H}_{4} \mathbf{B T A}$; this difference in free energy of binding $\left(\Delta G^{\mathrm{o}}\right.$ bind, $\mathbf{x - F y B T A}-\Delta G^{\mathrm{o}}$ bind, $\mathrm{H}_{\mathrm{BBTA}}=\Delta \Delta G^{\mathrm{o}}$ bind, Fluorination) is $1.1 \pm 0.3 \mathrm{kcal} \mathrm{mol}^{-1}$ more favorable (Figure $\mathrm{S} 1$ ). The increased binding affinity of $6,7-\mathbf{F}_{2}$ BTA over $\mathbf{H}_{4}$ BTA cannot be attributed to differences in buried hydrophobic area upon ligand binding (Figure S1), but arises from a more favorable entropy of binding. There are two plausible explanations for this increased binding affinity: i) the reorganization of the waters in the active site of HCA upon binding of the ligand are responsible

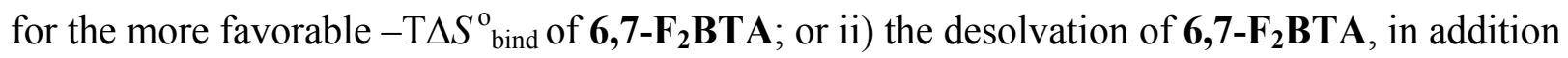
to the desolvation of the active site of HCA, influences the $\Delta G^{\mathrm{o}}$ bind.

Entropy-driven binding is compatible with the mechanism of the hydrophobic effect proposed by Kauzmann and Tanford, but is the only thiazole-based ligand studied thus far that is not enthalpy-dominated. The contribution of the enthalpy $\left(\Delta H^{\mathrm{o}}\right.$ ow $)$ and entropy $\left(-\mathrm{T} \Delta S^{\mathrm{o}}\right.$ ow $)$ of partitioning of $\mathbf{H}_{4}$ BTA and 6,7-F $\mathbf{F}_{2}$ BTA to the free energy of partitioning $\left(\Delta G^{0}\right.$ ow) mirrors the contributions of $\Delta H^{\mathrm{o}}$ bind and $-\mathrm{T} \Delta S^{\mathrm{o}}$ bind in the binding of these ligands to HCA (Figure S1).

WaterMap predicts $6,7-F_{2}$ BTA to be roughly equientropic with $\mathbf{H}_{4}$ BTA. WaterMap only accounts for first-order water correlation terms and only considers regions of high water density for the thermodynamic calculations and not for desolvation of the ligand. The most plausible explanation for this mirroring of trends of partitioning and binding is therefore that the 
desolvation of the ligand, and not just the desolvation of the active site of HCA, influences the $\Delta G_{\text {bind }}^{\mathrm{o}}$

The HS compensation observed in the binding of $4-F_{1} B T A$ and 4,6-F $\boldsymbol{B T A}$ arise from a reorganization of the solvent. The values of $\Delta H^{\circ}{ }_{\text {bind }}$ and $-\mathrm{T} \Delta S^{\mathrm{o}}{ }_{\text {bind }}$ of $4-\mathbf{F}_{\mathbf{1}} \mathbf{B T A}$ and $\mathbf{4 , 6}-\mathbf{F}_{2} \mathbf{B T A}$ are significantly different than $\mathbf{H}_{4}$ BTA $\left(>4 \mathrm{kcal} \mathrm{mol}^{-1}\right)$, but compensate and result in unchanged binding affinities. Interestingly, these ligands are the only two that rotate within the active site (Figure 2), possibly to reduce the unfavorable interaction of the fluorine at the 4-position with the backbone carbonyl of Thr 200 if the pose did not undergo rotation. The conserved binding of these ligands to HCA, and the conserved structure of the side chains of the amino acids of HCA in the active site suggest that neither changes in structure nor interaction of the protein and ligand are plausible candidates for significant changes in the thermodynamics binding. The most plausible candidate for the source of these compensated changes in $\Delta H^{\mathrm{o}}$ bind and $-\mathrm{T} \Delta S^{\mathrm{o}}$ bind is therefore the networks of hydrogen bonds of waters in the active site, and surrounding the ligands in solution and in the protein-ligand complex. WaterMap predicts the same trends for the observed thermodynamic of binding; the changed networks of water of 4-F BTA and 4,6F $_{2}$ BTA in the active site of HCA clearly indicate the importance of water in $H / S$ compensation (Figure 5). 


\section{Conclusions and interpretations}

The results obtained from the thermodynamic analysis, X-ray crystal structures, and molecular dynamics simulations described in this work show that a series of ligands with different electronic structure bind to HCA with very similar values of $\Delta G^{\mathrm{o}}$ bind, but with very

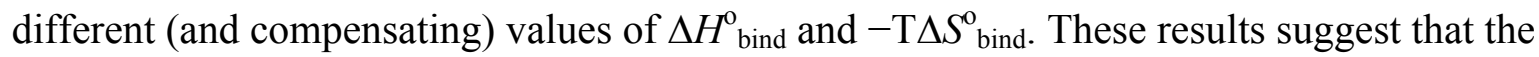
"size" (a term we cannot presently disaggregate into surface area, molecular volume, or dipole moment) of the ligand, and thus the water that is displaced from, or perturbed in the active site of HCA, is primarily responsible for the $\Delta G^{\mathrm{o}}$ bind; it also implies that changes in the structure of the networks of hydrogen bonded waters, that result upon ligand binding, determine the values of $\Delta H^{\mathrm{o}}{ }_{\text {bind }}$ and $-\mathrm{T} \Delta S^{\mathrm{o}}{ }_{\text {bind. }}$ This water-centric view of ligand binding-and $H / S$-compensationcannot be rationalized by the lock-and-key principle, and suggest that the molecules of water surrounding the ligand and filling the active site of a protein are as important as the structure of the ligand and the surface of the active site. 


\section{Acknowledgements}

The authors acknowledge Drs. Demetri T. Moustakas, Philip W. Snyder, and Dmitrji Rappoport, and Mr. Jang Hoon Yoon for their technical contributions and useful conversations. This work was supported by the National Science Foundation (NSF CHE-1152196) and the Wyss Institute for Biologically Inspired Engineering. M. A. thanks the Arab Fund Fellowship Program (AFFP) and the American University of Sharjah (UAE)for visiting scholar stipend award. H. L. thanks the German Research Foundation (DFG) for a postdoctoral research stipend.

\section{Author Contributions}

B.B., M.R.L. and G.M.W. designed research; B.B., M.R.L, W.S., S.F., M.A. H.L, C.M.B., and A.H. performed research; B.B., M.R.L, W.S., S.F., M.A. H.L, C.M.B., and A.H. analyzed data; and B.B, M.R.L., W.S., and G.M.W. wrote the paper.

\section{Competing financial interests}

The authors declare no competing financial interests.

\section{Additional Information}

Supplementary information is available online at online at http://www.nature.com/naturechemicalbiology. Reprints and permissions information is available online at http://npg.nature.com/reprintsandpermissions/. Supplementary information details the experimental procedures for the synthesis of the ligands, the purification of protein, the measurement of the thermodynamics of binding and partitioning, the measurement of the $\mathrm{pKa}$ of the ligands, the preparation and crystallography of the protein-ligand complexes, and the molecular dynamics calculation for the HCA-ligand complexes. Correspondence and requests for materials should be addressed to G.M.W. 


\section{Figure Legends}

Figure 1: (a) Structures of the partially fluorinated ligands used in this study, and their abbreviations. The abbreviation of each ligand indicates the number of fluorine atoms on the benzo-extension (e.g., $\mathbf{F}_{2}$ BTA contains two fluorine atoms). (b) An overlay of the heavy atoms of the $\mathbf{H}_{4}$ BTA and $\mathbf{F}_{4}$ BTA ligand from (aligned) crystal structures of the two HCA-ligand complexes. (c) Diagram of the amino acid residues of HCA that form contacts with the benzothiazole $\left(\mathrm{H}_{\mathbf{4}}\right.$ BTA) and per-fluorobenzothiazole (F $\mathbf{5}$ BTA) sulfonamide ligands, determined previously from crystal structures of each complex. Favorable interactions between the ligand and the protein are represented with a blue dashed line, and unfavorable interactions with a red dashed line.

Figure 2. Crystal structures of the active site of HCA complexed with: (a) 4-F $\mathbf{E}$ BTA, (b) 7 -

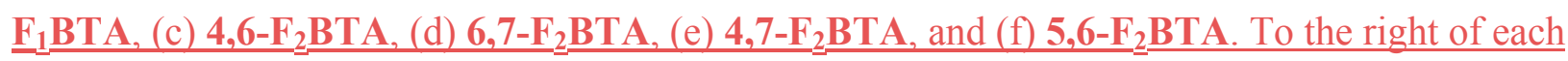
$\underline{\text { crystal structure is an overlay of the heavy atoms of the ligand with } \mathbf{H}_{4}} \underline{\mathbf{B T A} \text { or } \mathbf{F}} \underline{\underline{\mathbf{B T A}}} \underline{\mathbf{A} \text { from }}$

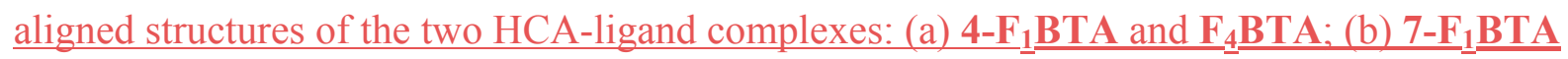

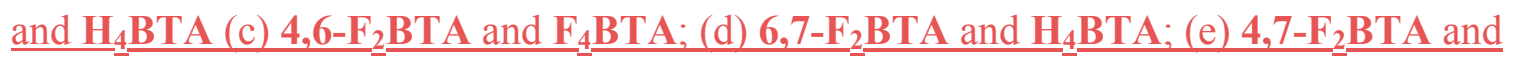

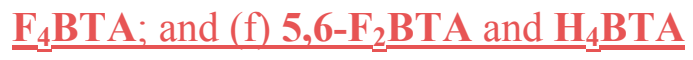

Figure 3 Diagram of the $\mathrm{p} K_{\mathrm{a}}$-corrected thermodynamic results $\Delta \Delta J^{\mathrm{o}}$ bind $\left(\right.$ compared to $\left.\mathbf{H}_{4} \mathbf{B T A}\right)$ where $J=G$ (blue), $H$ (green), and $S$ (red)—obtained from ITC measurements. The relative differences in the enthalpy and entropy of binding (i.e., mutual $H / S$ compensation) result in

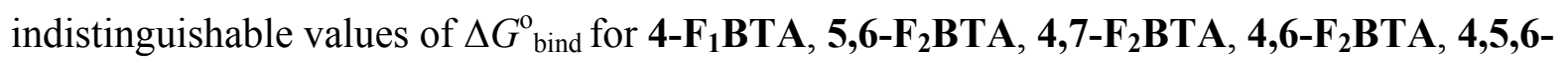


F $_{3}$ BTA, and F $_{4}$ BTA. The gray region demarcates the $95 \%$ confidence interval (i.e., two standard deviations) of $\Delta \Delta G^{\mathrm{o}}$ bind for $\mathbf{H}_{4}$ BTA.

Figure 4. Diagram comparing of the $\mathrm{p} K_{\mathrm{a}}$-corrected thermodynamics of $\Delta J^{\mathrm{o}}$ bind results from ITC measurements with WaterMap calculations for $\Delta G^{\mathrm{o}}$ bind $(\mathrm{a}), \Delta H_{\text {bind }}(\mathrm{b})$, and -T $\Delta S_{\text {bind }}^{\mathrm{o}}(\mathrm{v})$.

Figure 5. Hydration site thermodynamics for $\mathbf{H}_{4}$ BTA and three fluorinated variants $(\mathbf{4 , 6}-$ F $_{2}$ BTA, 5,6-F 2 BTA, and 6,7-F 2 BTA). Hydration site colors range from green (favorable) to red (unfavorable). The range for $\Delta H^{\mathrm{o}}{ }_{\text {bind }}$ (left panel) is $\left[-5.0\right.$ to $\left.+5.0 \mathrm{kcal} \mathrm{mol}^{-1}\right]$ whereas for $-\mathrm{T} \Delta S^{\mathrm{o}}{ }_{\text {bind }}$ (right panel) the range is $\left[0.0\right.$ to $\left.+5.0 \mathrm{kcal} \mathrm{mol}^{-1}\right]$. WaterMap computes all values relative to bulk

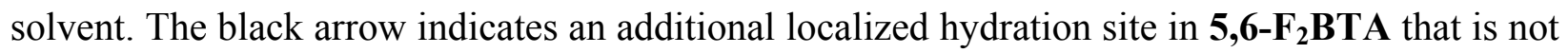
observed in other $\mathbf{x}-\mathbf{F}_{2}$ BTA variants. The dashed oval indicates a cluster of hydration sites that is

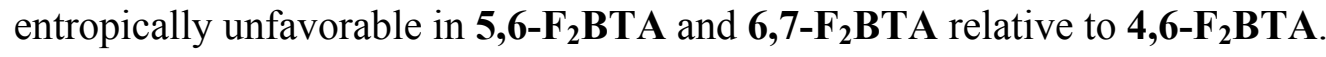




\section{References}

1 Biela, A. et al. Dissecting the hydrophobic effect on the molecular level: the role of water, enthalpy, and entropy in ligand binding to thermolysin. Angew. Chem., Int. Ed. 52, 1-8 (2013).

2 Lockett, M. R. et al. The Binding of Benzoarylsulfonamide Ligands to Human Carbonic Anhydrase is Insensitive to Formal Fluorination of the Ligand. Angew. Chem., Int. Ed. accepted (2013).

3 Snyder, P. W., Lockett, M. R., Moustakas, D. T. \& Whitesides, G. M. Is it the shape of the cavity, or the shape of the water in the cavity? Eur. Phys. J. Special Topics, doi:http://dx.doi.org/10.1140/epjst/e2013-01818-y (2013).

4 Snyder, P. W. et al. The origin of the hydrophobic effect in the biomolecular recognition of arylsulfonamides by carbonic anhydrase. Proc. Nat. Acad. Sci., U.S.A. 108, 1788917894 (2011).

5 Biela, A. et al. Ligand Binding Stepwise Disrupts Water Network in Thrombin: Enthalpic and Entropic Changes Reveal Classical Hydrophobic Effect. J. Med. Chem. 55, 60946110 (2012).

6 Chervenak, M. C. \& Toone, E. J. A direct measure of the contribution of solvent reorganization to the enthalpy of binding. J. Am. Chem. Soc. 116, 10533-10539 (1994).

7 Wilfong, E. et al. A multidisciplinary approach to probing enthalpy-entropy compensation and the interfacial mobility model. J. Am. Chem. Soc. 133, 11515-11523 (2011).

8 Abel, R. et al. Contribution of explicit solvent effects to the binding affinity of smallmolecule inhibitors in blood coagulation factor serine proteases. ChemMedChem 6, 10491066 (2011).

9 Abel, R., Young, T., Farid, R., Berne, B. \& Friesner, R. Role of the active-site solvent in the thermodynamics of factor Xa ligand binding. J. Am. Chem. Soc. 130, 2817-2831 (2008).

10 Salonen, L. M., Ellermann, M. \& Diederich, F. Aromatic Rings in Chemical and Biological Recognition: Energetics and Structures. Angew. Chem., Int. Ed. 50, 4808-4842 (2011).

11 Sharp, K. Entropy-enthalpy compensation: Fact or artifact? Protein Sci. 10, 661-667 (2001).

12 Chodera, J. D. \& Mobley, D. L. Entropy-enthalpy compensation: Role and ramifications in biomolecular ligand recognition and design. Ann. Rev. Biophys. 42, 121-142 (2013).

13 Dunitz, J. D. Win some, lose some - enthalpy-entropy compensation in weak intermolecular interactions. Chem. Biol. 2, 709-712 (1995).

14 Searle, M. S., Westwell, M. S. \& Williams, D. H. Application of a generalized enthalpyentropy relationship to binding cooperativity and weak associations in solution. J. Chem. Soc., Perkin Trans. 2 2, 141-151, doi:10.1039/p29950000141 (1995).

15 Lafont, V. et al. Compensating enthalpic and entropic changes hinder binding affinity optimization. Chem. Biol. Drug Des. 69, 413-422 (2007).

16 Qian, H. Entropy-enthalpy compensation: Conformational fluctuation and induced fit. $J$. Chem. Phys. 109, 10015-10017, doi:10.1063/1.477669 (1998). 
17 Qian, H. \& Hopfield, J. J. Entropy-enthalpy compensation: Perturbation and relaxation in thermodynamic systems. J. Chem. Phys. 105, $9292-9298$ (1996).

18 Grunwald, E. \& Steel, C. Solvent reoganization and thermodynamic enthalpy-entropy compensation. J. Am. Chem. Soc. 117, 5687-5692, doi:10.1021/ja00126a009 (1995).

19 Lumry, R. \& Rajender, S. Enthalpy-entropy compensation phenomena in water solutions of proteins and small molecules: a ubiquitous property of water. Biopolymers $\mathbf{9 , 1 2 1 5 -}$ 1227 (1970).

20 Rekharsky, M. V. et al. A synthetic host-guest system achieves avidin-biotin affinity by overcoming enthalpy-entropy compensation. Proc. Nat. Acad. Sci., U.S.A. 104, 2073720742 (2007).

21 Kauzmann, W. Physical chemistry of proteins. Ann. Rev. Phys. Chem. 8, 413-438 (1957).

22 Tanford, C. The hydrophobic effect and the organization of living matter. Science 200, 1012-1018 (1978).

23 Krishnamurthy, V. M. et al. Carbonic anhydrase as a model for biophysical and physicalorganic studies of proteins and protein-ligand binding. Chem. Rev. 108, 946-1051 (2008).

24 Tellinghuisen, J. \& Chodera, J. D. Systematic errors in isothermal titration calorimetry: concentrations and baselines. Anal. Biochem. 414, 297-299 (2011).

25 Krishnamurthy, V. M. et al. Thermodynamic parameters for the association of fluorinated benzenesulfonamides with bovine carbonic anhydrase II. Chem. Asian J. 2, 94-105 (2007).

26 Rasaiah, J., Garde, S. \& Hummer, G. Water in nonpolar confinement: From nanotubes to proteins and beyond. Ann. Rev. Phys. Chem. 59, 713-740 (2008).

27 Acharya, K. R. \& Lloyd, M. D. The advantages and limitations of protein crystal structures. Trends Pharmacol. Sci. 26, 10-14 (2005).

28 Young, T., Abel, R., Kim, B., Berne, B. J. \& Friesner, R. A. Motifs for molecular recognition exploiting hydrophobic enclosure in protein-ligand binding. Proc. Nat. Acad. Sci., U.S.A. 104, 808-813 (2007).

29 Yang, Y., Lightstone, F. C. \& Wong, S. E. Approaches to efficiently estimate solvation and explicity water energetics in ligand binding: the use of WaterMap. Expert Opin. Drug Discovery 8, 277-287 (2013).

30 Lazaridis, T. Inhomogeneous fluid approach to solvation thermodynamics. 2. Applications to simple fluids. J. Phys. Chem. B 102, 3542-3550 (1998).

31 Lazaridis, T. Inhomogeneous fluid approach to solvation thermodynamics. 1. Theory. $J$. Phys. Chem. B 102, 3531-3541 (1998). 